\title{
XXII.
}

\section{Ueber die Veränderungen der kleinen Arterien bei Nieren-Erkrankungen.}

(Aus der pathologisch-anatomischen Anstalt des städtischen Kankenhauses am Urban. Prosector Professor Dr. Carl Benda.)

Von

cand. med. Ulrich Friedemann in Berlin.

(Hierzu Tafel XIV.)

Schon sehr lange ist es bekannt, dass bei der chronischen interstitiellen Nephritis Veränderungen in den kleinsten KörperArterien auftreten. Ueber den histologischen Charakter dieser Veränderungen jedoch und ihr ursächliches Verhältniss zur NierenErkrankung sind die Meinungen noch hente getheilt. Neigt auch die überwiegende Mehrzahl der Autoren der Ansicht zu, dass in einer grossen Zahl der Fälle die Arterien-Veränderungen nicht die Ursache der Nieren-Erkrankung sind, sondern eine Folge derselben, oder dass beide durch dieselben Momente bedingt sind, so wird doch die Frage dadurch sehr complicirt, dass auf der anderen Seite Arteriosklerose und Atherom der Nieren-Arterien häufig zur Nierenschrumpfung führen und histologisch eine Unterscheidung zwischen den primären und secundären Gefäss-Veränderungen gewöhnlich nicht gemacht wurde. Zwar finden wir in den über diesen Gegenstand erschienenen zahlreichen Schriften neben den Veränderungen, die zweifellos arterioskleoretischer Natur sind, auch solche beschrieben, die einen etwas abweichenden Typus zeigen. Da man jedoch beide Arten von Veränderungen oft in derselben Niere, ja in demselben Gefäss fand, wurden sie nur als verschiedene Stadien desselben Processes aufgefasst. Da nun alle diese Untersuchungen nach älteren Methoden ausgeführt sind, so wandte ich bei den vorliegenden Arbeiten auf Rath des Herrn Professor Benda die Unna-Tänzer'sche Orcëinfärbung und die Weigert'sche Elastinfärbung an. Die 


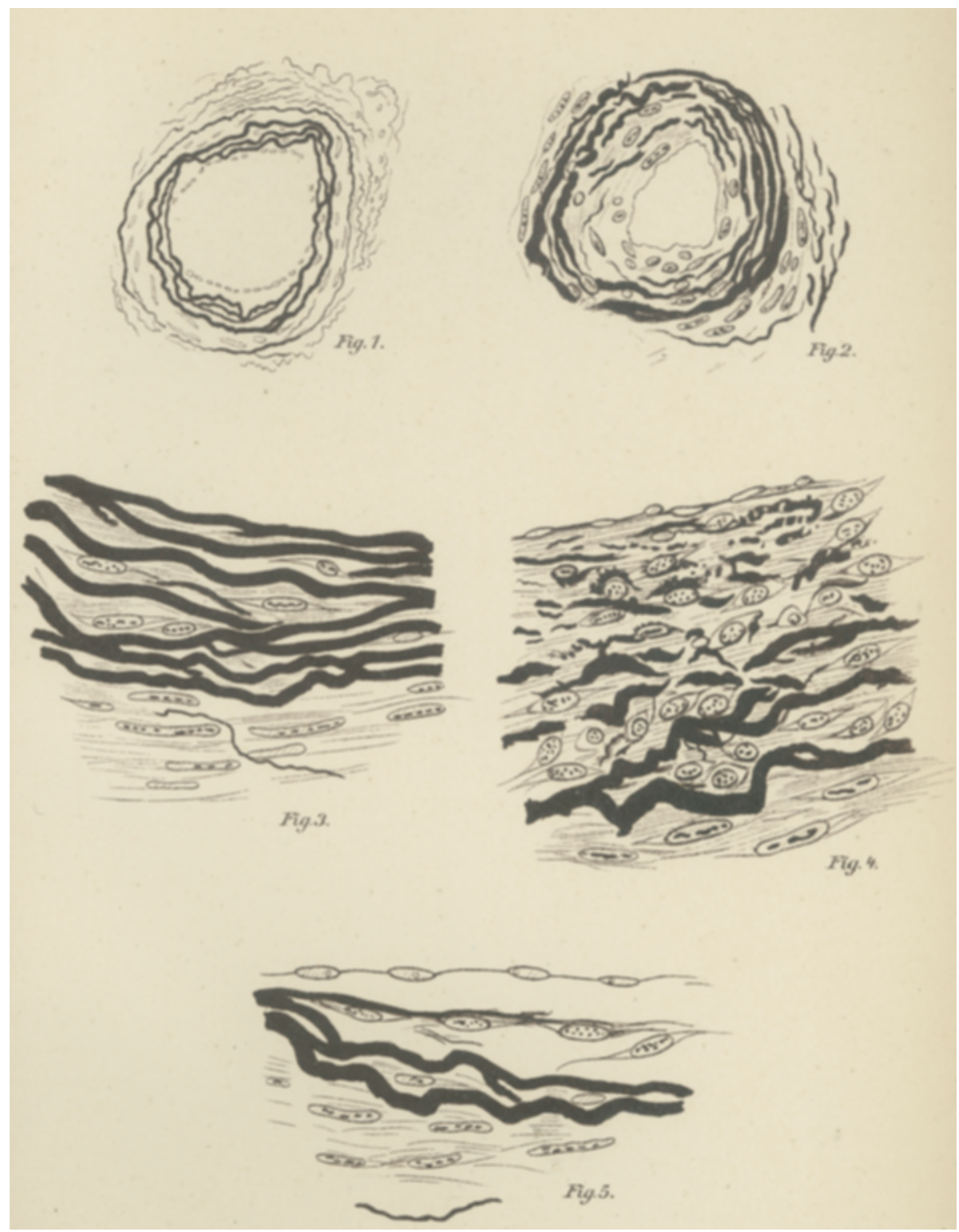


damit gewonnenen Resultate werde ich im Folgenden mittheilen und vor Allem untersuchen, welches der histologische Charakter der bei der interstitiellen Nephritis auftretenden Gefäss-Veränderungen ist, und in wie weit sie der Arteriosklerose und den übrigen Formen der Endarteriitis gegenüber eine selbständige Stellung einnehmen.

Da ich in vielen Punkten die Angaben der früheren Autoren bestätigt fand, so möchte ich zunächst auf ihre Befunde in Kürze eingehen. Der erste, der Gefäss-Veränderungen bei der Schrumpfniere constatirte, war Johnson, der in den kleineren KörperArterien eine Hypertrophie der Muscularis beobachtete. Ihm gegenüber betonten Gull und Sutton, welche die Gefässe der Pia mater untersuchten, mehr die Veränderungen in Intima und Adventitia. Sie beobachteten hier, bei Anwendung ibrer Methode (Färbung in Carmin, Aufbewahrung in Glycerin und Kampferwasser), das Auftreten glänzender Massen, die theils ein hyalines, theils ein fibröses Aussehen hatten. Kerne waren in diesen Massen nur spärlich vorhanden. Die fibrösen Massen sollten hauptsächlich bei älteren Individuen vorkommen. Die Media fanden sie nicht durchweg verdickt, vielmehr war sie an einzelnen Gefässen stellenweise sehr verdünnt, ja ganz geschwunden. Diese Veränderungen fanden sich bei demselben Individuum keineswegs in allen Gefässen, und auch nicht überall in gleicher Stärke. Später wurden diese Untersuchungen von Thoma nachgeprüft, der in den Nierengefässen ganz ähnliche Resultate gewann. Er fand in den Glomeruli hyaline Degeneration und Verödung der Gefässe, in den kleineren Nierengefässen ebenfalls hyaline Massen in der Intima, die in den grösseren Gefässen ein mehr fibröses Ausseben annahmen; in den noch grösseren Gefässen gesellten sich dazu Vascularisation und Atherom. Vor allem legte er darauf Gewicht, dass das Lumen in den meisten Fällen nicht durch diese Wandverdickung verengt war. In einzelnen Gefässen allerdings fand sich eine so starke Verdickung der Intima, dass das Lumen fast völlig verlegt war, und dann waren auch Vascularisation und Degenerations-Erseheinungen vorhanden.

Gleichzeitig mit diesen Untersuchungen erschien eine Arbeit von Ewald, der als erster eine Unterseheidung zwischen dem Atherom und den durch die Granularatrophie bedingten Ver- 
änderungen macht. Allerdings schliesst er etwas willkürlich bei allen Individuen unter 50 Jahren das Atherom aus. In diesen Fällen nun fand er an den Gefässen der Pia mater lediglich eine Hypertrophie der Muscularis, während er die von Gull und Sutton gefundenen Veränderungen in der Adventitia für Kunstproducte erklärt. Die von Thoma in den Nierengefässen gefundene "Endarteriitis fibrosa" hat nach seiner Ansicht nichts zu thun mit den Veränderungen der Piagefässe und stellt eine rein locale Erscheinung dar, die der Bindegewebsneubildung in der Niere selbst vollkommen analog ist.

Ich habe in der Hauptsache nur die Gefässe der Niere selbst, in einer geringen Zahl von Fällen auch die der anderen Organe, z. B. der Pia mater, untersucht und will daher aus eigener Anschauung über die von Ewald an diesen Gefässen gemachten Erfahrungen kein Urtheil füllen; ich glaube jedoch aus den Befunden an den Nierenarterien allein schliessen zu können, dass man nicht berechtigt ist, die in ihnen gefundenen Veränderungen von denen der anderen Gefässe aetiologisch abzutrennen. Ausserdem scheint es mir, dass Ewald den Intimaveränderungen an den Piagefässen nicht immer Beachtung schenkte, oder sie unberechtigter Weise dem Atherom zuzählte; haben doch Gull und Sutton an denselben Gefässen in einer ganzen Reihe von Fällen und bei zwei Individuen unter 50 Jahren, für die doch $\mathrm{Ewald}$ das Atherom ausschliessen zu können glaubt, Intima-Veränderungen beschrieben, die jedenfalls keine Kunstproducte sein können. Wenn ich nun auch zugeben will, dass die Veränderungen in den Piagefässen bisweilen einen etwas anderen Charakter haben können, so muss ich doch die später zu erweisende Behauptung vorausschicken, dass sie auf dieselben Ursachen zurückzuführen sind, wie die Veränderungen in den Nierengefässen.

Der genaueren histologischen Untersuchung stellten sich nun einige technische Schwierigkeiten in den Weg. Da die Schnitte durch das Parenchym der Niere gelegt wurden, so musste ich natürlich darauf verzichten, von allen Gefässen wirkliche Querschnitte zu erhalten, die doch für die Beurtheilung der Dickenverhältnisse eines Gefässes von grosser Wichtigkeit sind; ich habe jedoch diesen Uebelstand dadurch zu beseitigen Archiv f. path. Anat. Bd. 159. Hft. 3. 
gesucht, dass ich die Schnitte möglichst parallel zur Oberfläche legte und auf diese Weise wenigstens von den Arteriae interlobulares und Arteriolae rectae Querschnitte erhielt. In einzelnen Fällen habe ich auch kleine Arterien herauspräparirt und von ihnen Querschnitte angefertigt. Wie ich im Einzelnen den Schiefschnitt auszuschalten suchte, wird weiter unten erörtert werden.

Die Theile wurden meist der Leiche gleich nach dem Tode entnommen und in Formalin, theilweise auch in Sublimat gehärtet, was aber in Bezug auf die Resultate keinen Unterschied machte. Zur Färbung benutzte ich Orcëin und Methylenblau, sowie den Weigertschen Farbstoff, wobei ich mit Lithioncarmin oder nach van Gies on nachfärbte. Namentlich die letztere Methode giebt sehr gute Bilder, da sie die elastische Substanz, Bindegewebe, Kerne und Zellen in verschiedener Färbung hervortreten lässt. Daneben wurden auch Schnitte mit Hämatoxylin und Eosin nach van Gieson behandelt.

Genauer untersucht habe ich 19 Nieren und zwar zunächst ohne Rücksicht auf die Art der Nieren-Erkrankung; in 17 fanden sich Gefäss-Veränderungen, nur in 2 Fällen fehlten dieselben. Der eine dieser Fälle betraf eine frische parenchymatöse, der andere eine chronische parenchymatöse Nephritis. Ich habe noch mehrere Fälle von frischer parenchymatöser Nephritis auf das Verhalten der Gefässe geprüft und in ihnen niemals Veränderungen feststellen können. In den anderen Fällen fanden sich mehr oder weniger starke interstitielle Veränderungen, und zwar bot ein Theil der Fälle das Bild der genuinen Schrumpfniere, während andere Nieren als grosse weisse Nieren mit beginnender Schrumpfung bezeichnet werden mussten. Auch in diesen fanden sich Veränderungen der kleinsten Nierengefässe, doch waren meist nur einzelne Arterien von der Veränderung ergriffen. Ein Fall machte von dem geschilderten Verhalten eine Ausnahme, indem die sehr starken Gefäss-Veränderungen nicht von entsprechenden interstitiellen Processen in der Niere begleitet waren. Dieser Fall wird weiter unten seine Erklärung finden.

An den mit Hamatoxylin-Eosin oder nach van Gieson behandelten Präparaten konnte ich nun die Beobachtungen von Thoma vollauf bestätigen. In der Adventitia finden sich die 
streifigen oder homogenen, kernarmen Massen, die Media ist im Allgemeinen verdickt, an einzelnen Stellen atrophisch oder vollkommen fehlend, in der Intima findet sich dem Anschein nach eine fibröse oder hyaline Verdickung. Dabei ist das Gefäss theils in seinem ganzen Umfang verdickt, theils betrifft die Dickenzunahme nur einzelne Stellen. Im Allgemeinen ist die Intima in den kleinsten Arterien im Vergleich zum Lumen ungleich stärker verdickt, als in den etwas grösseren Gefässen. In einzelnen anderen Fällen sind aber gerade die grösseron Gefässe auffallend stark verdickt and zeigen neugebildete Vasa vasorum, sowie nekrotische Heerde in der Intima.

Schon die vielfachen Widersprüche in dieser Beschreibung legen die Vermuthung nahe, dass es sich hier offenbar nicht um einen einheitlichen Process handelt. Mit grösserer Sicherheit liess sich jedoch in den mit Orcëin und dem Weigert'schen Farbstoff gefürbten Präparaten erkennen, dass hier wirklich zwei aetiologisch verschiedene Veränderungen vorliegen, von denen die eine in ursächliche Beziehung zur Nieren-Erkrankung zu bringen ist, während die andere mit der Arteriosklerose identisch ist und in keinem directen Zusammenhange mit dem Process in der Niere zu stehen braucht. In den ausgesprochenen Fällen ist eine Trennung histologisch sehr wohl möglich, dagegen war es im Einzelnen oft recht schwierig $7 u$ entscheiden, welche Veränderungen auf die Nierenerkrankung allein, welche auf die Arteriosklerose zurückzuführen sind. Denn selbstverständlich kann sich der Einfluss der Nieren-Erkrankung mit dem der Arteriosklerose in jedem Verhältnis combiniren, was um so mehr ins Gewicht fällt, als die zur Section kommenden Schrumpfnieren meist Personen des höheren Alters betreffen, bei denen sich neben der interstitiellen Nephritis auch Arteriosklerose findet. Wir müssen also darauf gefasst sein, neben den scharf abgrenzbaren Fällen alle möglichen Uebergangsstufen zu finden. Von grosser Wichtigkeit war es daher, dass ich durch die Liebenswürdigkeit des Herrn Geheimrat Arnold einen Fall (Fall 1) untersuchen konnte, der ein noch ziemlich jugendliches Individuum von 38 Jahren betraf, bei dem sich an den grösseren Gefässen keine Spur von Arteriosklerose fand. Auf Grund dieses Falles war es mir dann möglich, in den meisten Fällen zu entscheiden, 
ob die Veränderungen allein durch die Nierenschrumpfung, oder auch durch die Arteriosklerose bedingt waren. Ich wende mich zunächst zur Beschreibung derjenigen Veränderungen, bei denen die Arteriosklerose auszuscheiden ist.

Wendet man die specifische Färbung für die elastische Substanz an, so sieht man, dass die in den gewöhnlichen Präparaten als hyaline oder fibröse Substanz impovirenden Verdickungen der Intima durch geschichtete elastische Membranen, die mit Orcëin gefärbt braun, bei der Weigert'schen Färbung tief blauschwarz erscheinen, gebildet sind. Ebenso sind die glänzenden Massen in der Adventitia wesentlich durch ein Netz elastischer Fasern gebildet. Die "fibröse Endarteriitis" von Tho ma, ebenso wie die von Gull und Sutton gefundene "Arterio-fibrosis", ist also das Product einer durch die Färbungsmethode entstandenen Täuschung und existirt thatsächlich nicht. Dass es sich in der Intima wirklich um Membranen handelt, beweisen Längsschnitte, auf denen sie ebenfalls als continuirliche Linien erscheinen, und Schrägschnitte, in denen man bei verschiedener Tubus-Einstellung die Membranen direct zu Gesicht bekommt. Betrifft die IntimaVerdickung gleichmässig den ganzen Umfang des Gefässes, was ich aber in den wenigsten Fällen sah, so verlaufen die Membranen concentrisch, sonst verzweigen sie sich auf der einen Seite, um sich auf der anderen wieder zu vereinigen. Oft spalten sich auch Membranen ab, die frei in der Gefässwand enden. Im Allgemeinen sind die Membranen gleich stark, nur die innersten sind bisweilen etwas dünner und weniger geschlüngelt, als die äusseren. Sie sind scharf contourirt und zeigen keineswegs ein gezacktes oder körniges Aussehen (Fig. 1 u. 3). Das Endothel liegt im Querschnitt als dünnes Hüutchen über der innersten Membran und zieht über deren Wellen hinweg. Nur an ganz vereinzelten Stellen konnte ich zwischen Endothel und Elastica noch einige spindelförmige Zellen beobachten. In den Maschenräumen der elastischen Membranen befindet sich eine spärliche Grundsubstanz, in der Zellen eingebettet sind. Die Zellen haben einen ovalen oder spindelförmigen Kern, einen länglichen oder polygonalen Zellenleib und scheinen in Hohlräumen zu liegen, die sie nicht ganz ausfüllen. Zwischen zwei elastischem Membranen liegt stets nur eine Zellschicht. Die Media ist ebenfalls 
im Ganzen verdickt, jedoch gewöhnlich nicht in dem Maase, wie wir es später bei der Arteriosklerose seben werden. Dabei sieht man, dass auch die Zahl der Muskelkerne vermehrt ist, Sitzt die Intima-Verdickung einseitig, so ist auch die Media oft über dieser Stelle verstärkt. Bisweilen ist sie hier aber auch geschwunden, so dass sich die elastischen Membranen auf Kosten der Media entwickelt $z u$ haben scheinen. In einzelnen Fällen von sehr starker Schrumpfniere fand sich in den kleineren Gefässen die Intima ringsherum sehr stark verdickt, während die Media ganz geschwunden war. Da ich bei den einseitig sitzenden Gefäss-Veränderungen auf den Verdacht gekommen war, es möchte sich hier um Täuschungen, die durch Schiefschnitte entstanden sind, handeln, so habe ich an herauspräparirten Stücken von kleinen Arterien in der Art Serienschnitte angefertigt, dass ich die Schnittrichtung variirte, und ich beobachtete, dass in einem kleinen Gefäss, das auf der verdickten Seite etwa fünf, auf der dünneren nur eine elastische Membran hatte, bei Veränderung der Schnittrichtung die Membranen zwar mehr als Flächensegmente erschienen, in ihrer Zahl sich jedoch nicht. änderten.

Wesentlich andere Verhältnisse bieten nun in Bezug auf das Gefässsystem die meist älteren Individuen angehörigen Nieren, bei denen sich von den grösseren Arterien her arteriosklerotische und atheromatöse Processe auf das Gefässsystem der Niere fortsetzen. Es finden sich dann die Veränderungen, die Thoma (dieses Archiv Bd. 71 Seite 244 u. 45) richtig beschrieben hat, die er jedoch nur als ein jugendlicheres Stadium der bisher beschriebenen Gefäss-Veränderungen auffasst. Betrachtet man nur die mit Hämatoxylin-Eosin oder nach van Gieson gefärbten Präparate, so erscheinen beide Processe allerdings ziemlich ähnlich, und es ist nicht zu verwundern, dass Thom a zu einer derartigen Auffassung kam. In Orcëin-Präparaten fällt jedoch sofort auf, dass veben Gefässen, welche die oben beschriebenen Veränderungen zeigen, andere sehr wesentliche Abweichungen aufweisen. Im Ganzen ist die Verdickung der Gefässwand und die Verengerung des Lumen eine weit mehr ausgeprägte. In der Intima sind ebenfalls viele neugebildete, gut gefärbte elastische Membranen zu sehen, ja ihre Zahl ist sogar durchschnittlich grösser, als bei den anderen Gefässen. Es zeigt sich aber, dass die Zwischen- 
substanz viel reichlicher ist. Vor Allem findet sich nach innen von den Membranen eine mehr oder weniger breite Schicht, in der das zellige und fibröse Element vorherrscht (Fig. 2). Zwar finden sich noch Andeutungen von elastischer Substanz, dieselbe färbt sich jedoch schlecht, besteht nur aus Fäserchen und Körnchen, und geht stellenweise diffus in die Umgebung über. Noch deutlicher werden diese Verhältnisse in den mit der Weigertschen Färbung behandelten Präparaten, welche die elastische Substanz weit deutlicher hervortreten lässt. Während die äusseren Membranen noch ziemlich intact sind, beginnen sie nach innen zu ein gezacktes, rosenkranz-ähnliches Aussehen anzunehmen, wie es Neumann und Weissmann und Dmitrijeff beschrieben haben. Weiterhin zerfallen sie in eine Reihe einzelner schwarzer Bröckel, und in der innersten Schicht findet man nur noch schwarze Körnchen, die um die Zellleiber herumliegen (Fig. 4). Die Zellen sind in der innersten Schicht ziemlich reichlich vorhanden. Daneben finden sich dann in einzelnen Gefässen Veränderungen, die schon ein weiteres Stadium bezeichnen: in der Intima nekrotische Heerde, Züge von Fibroblasten, welche die Gefässwand durchqueren und offenbar neu gebildete Vasa vasoram darstellen, kurzum alle die Veränderungen, die als charakteristisch für die Arterioklerose beschrieben sind.

Die Media ist ebenfalls sehr stark verdickt, doch ist eine dieser Verdickung entsprechende Vermehrung der Muskelkerne nicht $z u$ beobachten. Dieselben stehen ziemlich weit auseinauder, und eine Vergleichung mit einem normalen Gefäss gleichen Kalibers ergiebt, dass die Zahl der in der Media vorhandenen MuskelkernLagen nicht wesentlich vermehrt ist. Mit der van Giesonschen Färbung erkennt man, dass die Dickenzunahme der Media hauptsächlich auf eine Vermehrung des in derselben vorhandenen Bindegewebes zurückzuführen ist. Man darf nun keineswegs erwarten, dass die Arteriosklerose sich gleichmässig in den kleineren Nierenarterien localisirt. Vielmehr waren auch in den mit starker Arteriosklerose complicirten Fällen stets nur einige Gefässe in der angegebenen Art erkrankt, während die Mehrzahl die vorher bescliriebenen Veränderungen aufwies.

Die Verödang der Glomeruli ist ein Process, der von den beschriebenen vollkommen $z u$ trennen ist. Auch nach den 
Elastin-Färbungen handelt es sich hier um eine einfache hyaline Degeneration. Ein ganz ähnliches Verhalten zeigen bisweilèn die kleinsten Arterien, die schon den Uebergang zu Capillaren bilden, in sehr stark geschrumpften Nieren. Die Media ist sehr atrophisch, und in der Intima findet sich eine homogene Masse, in der wenige ovale Kerne, bisweilen auch 1 bis 2 elastische Membranen liegen.

Um dieselbe Art von Veränderungen handelte es sich wohl bei einem 24jährigen Individuum, bei dem die Nieren so starke Schrumpfung zeigten, dass von dem Parenchym nur noch kleine Reste vorhanden waren. Aus später zu erörterndenGründen sind die oben beschriebenen Gefäss-Veränderungen hier nicht vorhanden, dagegen ist in einzelnen Arterien die Elastica interna stark verdickt, ohne jedoch Auffaserung zu zeigen. Nach innen von dieser Membran liegt eine homogene Masse, in der wenige Kerne za sehen sind; hie und da sind auch Andeutungen von elastischen Membranen, die unter einander, aber nicht mit der Elastica interna zusammenhängen, vorhanden, doch sind dieselben nicht so stark gefärbt, und bei starker Vergrösserung sieht man, dass sie nicht continuirliche Bänder darstellen, sondern aus einzelnen Stücken zusammen gesetzt sind, für die immer eine Zelle den Kern abgiebt ${ }^{1}$ ). Wahrscheinlich ist diese Erkrankung mit der "Endarteriitis obliterans" identisch, die Heubner usprünglich für eine specifisch luische ansah, die aber, wie namentlich Baumgarten und Friedländer betonten, überall auftritt, wo grosse Theile des Verzweigungs-Gebietes einer Arterie zu Grunde gehen. Der grosse Unterschied von der zuerst beschriebenen Art von Veränderangen

1) Erst nach Abschluss meiner Untersuchungen ist mir die Arbeit von Jores (Ziegler's Bd. 24) „Ueber die Neulildung elastischer Fasern in der Intima bei Endarteriitis" zu Gesicht gekommen, so dass ich dieselbe nicht mehr berücksichtigen konnte. Ich fand dort in vielen Punkten meine Resultate bestätigt, doch bat Jores seine Untersuchungen an anderen Objecten vorgenommen und die bier behandelten Fragen nur gestreift. Hervorbeben möchte ich, dass Jores in unterbundenen Gefässen an der elastiseben Substanz Befunde gmacht bat, die mit den so eben beschriebenen grosse Aehnlichkeit haben. Wabrscheinlich bandelt es sich in diesem Fall um dieselbe Erscheinung, doch wäre es auch möglich, dass wir es hier mit Degeneration zu thun baben. 
besteht darin, dass bei der Endarteritis obliterans die zellige Wucherung das Wesentliche ist, während die Neubildung der elastischen Membran offenbar nur eine secundäre Anpassung an die veränderten Blutdruck-Verhältnisse darstellt.

Wir haben also in Schrumpfnieren drei verschiedene Arten von Gefässveränderungen beobachtet, von denen die Arteriosklerose als eine nicht nothwendigerweise zur Nierenkrankheit gehörige Complication anzusehen ist, die Endarteriitis obliterans sich nur spärlich und in den stärker erkrankten Nieren zeigt.

Nach den mitgetheilten Thatsachen ist es nun nicht schwer, sich für die zuerst beschriebenen Veränderungen ein Bild von dem Wesen des Processes zu machen. Entzündungs-Erscheinungen fehlen vollkommen, vor allem ist keine kleinzellige Infiltration und keine Gefäss-Neubildung vorhander.. Den Process als das Product einer abgelaufenen Entzündung aufzufassen, ist aber deshalb unzulässig, weil man dann doch in dem einen oder anderen Gefäss frische entzündliche Heerde finden müsste. Diese fehlen aber, wie bereits bemerkt, in den nicht arteriosklerotisch erkrankten Gefässen vollkommen. Ebenso, wie die EntzündangsErscheinungen, fehlen, selbst in den am stärksten veränderten Gefässen, jegliche Erscheinungen von hyaliner Degeneration oder Nekrose. Hält man damit zusammen, dass Media und Adventitia hypertrophisch gefunden wurden, so kann man auch den Process in der Intima getrost als eine Hypertrophie der elastischen Gewebs-Bestandtheile ansehen, bei der die Zellen nur eine vermittelnde Rolle spielen. Wir müssen also die die Schrumpfniere begleitenden Gefäss-Veränderungen, die Arterio-fibrosis von Gull und Sutton oder die Endarteritis von Thoma, als einfache Hypertrophie der Arterien bezeichnen.

Wodurch aber wird diese Hypertrophie der Gefässwand, und speciell der elastischen Gewebs-Bestandtheile derselben hervorgerufen? Die normale Function der Elastica interna ist es, der Wandspannung entgegenzuarbeiten und damit eine Dehnung oder Zerreissung der Wand zu verhüten. Die Spannung in einem Gefäss ist aber gleich dem Product aus Durchmesser und Druck, muss also steigen, wenn letzterer eine Zunahme erführt. Da wir nun wissen, dass die einfachen Hypertrophien der Gewebe meist auf eine erhöhte Thätigkeit derselben zurückzuführen sind, 
so können wir auch hier eine Drucksteigerung in den Gefässen für die Verdickung der Wand und die Vermehrung der elastischen Membranen verantwortlich machen, und wir kommen damit zu einem Schluss, den uns die klinischen Erscheinungen der Granularatrophie schon lange aufgedrängt haben. Dass die Verdickung meist nur einseitig vorhanden ist, findet wohl zum Theil dadurch seine Erklärung, dass an den nicht verdickten Stellen das umgebende Gewebe eine festere Stütze für die Gefässwand abgiebt und so eine stärkere Dehnung derselben verhindert wird. Vor allem dürfte aber hier die Schlängelung der Arterien in Betracht kommen, da an der convexen Seite die Gefässwand bei der Drucksteigerung weit stärker gespannt wird, als an der concaven.

Wie sie den histologischen Charakter der Gefäss-Veränderungen abweichend deuteten, so haben Gull und Sutton, sowie Thoma auch eine andere Auffassung von der Entstehung derselben. Gull und Sutton glaubten, dass es sich dabei um eine über das ganze Gefässsystem verbreitete hyaline Degeneration an den kleineren Gefässen handele, die den Schrumpfungs-Process in der Niere, sowie die bei der Schrumpfniere vorhandenen Veränderungen anderer Organe hervorrufe, - eine Behauptung, die jedoch allenthalben auf Widerspruch stiess. Vor Allem hielt man dieser Ansicht mit Recht entgegen, dass eine wirkliche Verengerung der Gefässe intra vitam gar nicht vorhanden sei (Thoma). Erst post mortem zieht sich durch den Fortfall des Blutdruckes das Gefäss zusammen, und es ist ersichtlich, dass diese Zusammenziehung eine besonders starke sein muss, wenn, wie bei der Nephritis interstitialis, die Elasticität der Gefässwand erhöht ist. Thoma nahm an, dass die durch die Schrumpfniere bedingte Verödung eines grossen Theils der Capillarbahn die Ursache der Gefäss-Veränderungen sei, und hielt dieselben für identisch mit der Endarteriitis obliterans, die $m a n$ in nekrotischen Gewebspartien, Geschwülsten, unterbundenen Gefässen u. s. w. findet. Diese soll aber direct durch eine Strom-Verlangsamung in den betreffenden Gefässen hervorgerufen werden. Diese Erklärung kann jedenfalls nur auf die hyaline Degeneration der Glomeruli und die oben erwähnte zellig-fibröse Endarteriitis der kleinsten Arterien Anwendung finden. Für die etwas grösseren Arterien, um die es sich hier und in den Arbeiten der erwähnten Autoren 
hauptsächlich handelt, ist sie nicht zutreffend. Denn erstens ist die Endarteriitis obliterans von der Hypertrophie der Arterien bei der Schrumpfniere histologisch vollkommen verschieden. Bei jener handelt es sich wirklich um die Neubildung eines mehr oder weniger zellenreichen Bindegewebes, während dieselbe bei der Arterien-Hypertrophie nur durch die Färbungsmethode vorgetäuscht wird. Zweitens ist eine Strom-Verlangsamung in geschrumpften Nieren meiner Ansicht nach nicht erwiesen. Thoma hat zwar an der Leiche entnommenen Schrumpfnieren bei einem Druck, der dem normalen Blutdruck gleichkommt, DurchströmungsVersuche gemacht and dabei eine Verlangsamung des FlüssigkeitsStromes gegenüber gesunden Nieren constatirt. Diesen Versuch halte ich jedoch nicht für beweisend, da der bei der Schrumpfniere vorhandene erhöhte Blutdruck docì offenbar lange Zeit im Stande ist, das Blut mit normaler Geschwindigkeit durch die Nieren zu treiben.

Thoma hat die Beziehungen zwischen den mechanischen Verhältnissen des Blutkreislaufs und dem Wachsthum der Gefässe in drei Gesetzen formulirt, deren zweites lautet: „Das Dickenwachsthum der Gefässwand ist abhängig von der Wandspannung." Merkwürdigerweise hat er von diesem Gesetz, dass sich bei den lier in Frage stehenden Veränderungen vollkommen bewährt hat, für die Pathologie fast gar keinen Gebrauch gemacht. Das Hauptgewicht legt er auf sein erstes histo-mechanisches Princip. „Das Wachsthum der Gefässlichtung, oder, was gleichbedeutend ist, das Flächenwachsthum ist abhängig von der Strom-Geschwindigkeit des Blutes u. s. w.", und dies hat wahrscheinlich darin seinen Grund, dass Thoma bei Anwendung der damals üblichen Methoden die Neubildung in der Intima der Arterien bei Schrumpfnieren, bei der Arteriosklerose, in den Arterien von Amputationsstümpfen und in der Aorta nach der Geburt für identische Processe ansah, und auch eine einheitliche Erklärung für dieselben geben wollte. Wie weit dieses Princip für die beiden letztgenannten Erscheinungen in Betracht kommt, kann ich hier nicht entscheiden. Jedenfalls scheint es mir für die vorliegenden Veränderungen und für die Arteriosklerose keine ausreichende Erklärung zu geben. Solange man die Neubildung in der Intima für Bindegewebe, also für ein indifferenzirtes Gewebe ansah, konnte 
man sich mit dieser Erklärung begnügen, wenn sie auch keine Vorstellung von dem Zusammenhang zwischen Bindegewebs-Neubildung und Strom-Geschwindigkeit giebt, sondern die Neubildung in der Intima nur als eine zweekmässige Einrichtung deutet (vergl. Thoma, Pathologische Anatomie). Bildet sich dagegen in der Intima ein mit einer specifischen Function begabtes Gewebe, wie das elastische, so sind wir nach den von Roux (vergl. Kampf der Theile im Organismus) entwickelten Principien im Stande, eine wirklich histo-mechanische Erklärung zu geben.

Die von Ew ald ausgesprochene Ansicht, dass die Veränderungen in der Intima der Nierengefässe den interstitiellen entzündlichen Processen in der Niere analog seien, kann schon darum nicht richtig sein, weil es sich dabei, wie erwähnt, gar nicht um Bindegewebs-Neubildung handelt.

Tür die Arteriosklerose giebt Th om a eine ähnliche Erklärung, wie für seine Endarteriitis fibrosa. Nach ihm tritt zunächst eine Schwächung der Elasticität der Media ein. In Folge dessen wird die Gefässwand gedehnt, das Lumen erweitert, und die Folge davon ist Strom-Verlangsamung, welche nun ihrerseits wieder das ursächliche Moment für die Neubildung in der Intima abgiebt. Durch diese wird das Lumen wieder auf seine normale Weite reducirt. Sehen wir daraufhin die an den kleineren Arterien gemachten Befunde bei der Arteriosklerose an, so müssen wir zunächst die schon oben constatirte massenhafte Zunahme der elastischen Membranen zwischen Intima und Media auf dieselben Ursachen zurückführen, wie bei der Arterien-Hypertrophie bei Schrumpfniere, d. h. auf die erhöhte Spannung der Gefässwand. Da wir diese Spannungs-Erhöhung bei der Arteriosklerose nicht auf eine Blatdruck-Steigerung zurückführen können, so bleibt uns zu ihrer Erklärung nur die Annahme, dass sich durch eine abnorme Dehnung der Gefässwand der Querschnitt vergrössert hat und dadurch die Elastica intema stärker gespanot wird. Dieser Schluss wird durch die experimentellen Arbeiten Thoma's bestätigt, der an arteriosklerotischen Arterien, noch ehe deutliche Veränderungen in der Intima vorhanden waren, eine Abnahme der Elasticität constatirte und diese auf die Media bezog. Das anatomische Substrat für diese Abnahme der Elasticität ist offenbar die schon erwähnte diffuse, wahrscheinlich mit hyaliner 
Degeneration verbundene Bindegewebs-Zunahme in der Media, die wahrscheinlich von einer Schwächung der Muskelfasern begleitet ist. In den von mir untersuchten Fällen ist die Vermehrung der elastischen Membranen wohl nicht allein auf die Arteriosklerose zurückzuführen, sondern zum Theil durch die gleichzeitig vorhandene Nephritis interstitialis bedingt. Doch haben Heubner, Langhans, Dmitriejeff u. a. auch bei reiner Arteriosklerose eine Vermehrung der elastischen Membranen beobachtet.

Wie aber sind die Veränderungen in der innersten Schicht der Intima zu deuten? Thoma trennte dieselbe nicht von den darunter liegenden elastischen Membranen, und hielt beide zusammen für eine compensatorische Verdickung. Ihr verschiedener anatomischer Charakter spricht aber schon dafür, dass sie auch verschiedenen Ursachen ihre Entstehung verdanken. Vor Allem der Umstand, dass in den innersten Schichten die DegenerationsErscheinungen an den elastischen Gebilden vorherrschen, hat mich zu der Ansicht gebracht, dass es sich bei der AltersArteriosklerose, (wenigstens in den von mir untersuchten kleineren Arterien), zunächst um eine diffuse Vermehrung des Bindegewebes mit Untergang der specifischen Gewebs-Elemente, wie sie ja für die Alters-Veränderungen auch in anderen Organen charakteristisch ist, in Intima und Media handelt. Dadurch nimmt die Elasticität der Wand $a b$, und das Gefäss würde gedehnt werden, wenn nicht durch eine Vermehrung der elastischen Membranen die normale Elasticität hergestellt würde.

Im Anschluss hieran muss ich noch einmal auf den schon erwähnten Fall zurückkommen, bei dem sich eine starke, reine Hypertrophie der Arterien ohne entsprechende Veränderungen in den Nieren fand. Auch Gull und Sutton beobachteten mehrere solcher Fälle, die einer Erklärung grosse Schwierigkeiten boten. Diese einfache Arterienhypertrophie ist schon von Langhans, Talma, Heubner und Dmitrijeff an den Gehirnarterien beschrieben worden. Sie wurde von ihnen jedoch einfach als eine Localisation des im übrigen Gefässsystems verbreiteten arteriosklerotischen Processes an den Hirnarterien betrachtet. In dem von mir und wahrscheinlich auch in den von den erwähnten Autoren untersuchten Fällen waren nun die Aorta und 
die grösseren Gefässe so stark atheromatös und arteriosklerotisch verändert, dass dieselben fast in starre Röhren verwandelt waren; die der Pulswelle jedenfalls nur sehr wenig nachgeben konnten. Dadurch wird sich aber letztere mit wenig verminderter Energie bis in die kleinsten Arterien fortpflanzen, wie dies ja auch klinisch durch das Pulsiren der kleinsten Arterien bei der Arteriosklerose erwiesen ist. Da aber die Elastica interna die Aufgabe hat, die durch die Pulswelle gedebnte Gefässwand wieder zur Zusammenziehung zu bringen, so wird die bei jeder Systole erhöhte Spannung einen Reiz zur Hypertrophie abgeben. Die Arteriosklerose der kleineren Arterien stellt also in vielen Fällen nur eine einfache Hypertrophie dar, die den durch die Arteriosklerose der grossen Gefässe gesetzten mechanischen Veränderungen des Blutkreislaufs ihre Entstehung verdankt. Ist die Arterien-Hypertrophie, wie wir gesehen haben, lediglich eine Folge der Granularatrophie, so kann die Arteriosklerose durch die Verengerung der Gefässlumina sehr wohl zur Nierenschrumpfung führen. Meiner Ansicht nach ist man jedoch nur berechtigt, diejenigen Schrumpfnieren als arteriosklerotische zu bezeichnen, in denen wirklich die Gefässe durch BindegewebsWucherung verengt sind, während die Arterien, die nur durch neugebildete elastische Membranen verdickt sind, mag diese Verdickung noch so hochgradig sein, dem Blutstrom nie ein wirkliches Hinderniss bieten können.

Im Vorhergehenden waren wir za dem Schluss gekommen, dass die Arterienhypertrophie bei der Schrumpfniere durch den erhöhten Blutdruck zu Stande kommt. Daraus folgt, dass sich diese Veränderungen auch in den kleinen Arterien der übrigen Organe finden müssen, und ich habe auch in einzelnen Fällen diese daraufhin untersucht. Der Verwerthung der Ergebnisse stellten sich jedoch grosse Schwierigkeiten entgegen, die aus dem Vorhergehenden verständlich sind. Da es sich meist um ältere Individuen handelte, die ich untersuchen konnte, so waren meistens die grossen Gefässe stark atheromatös erkrankt, oder die Veränderungen in den kleinen Arterien zeigten sogar einen arteriosklerotischen Charakter. Ich kann daher alle diese Fälle nicht als beweisend ansehen. Im Fall 1 fanden sich allerdings an den Gefässen der Pia mater keine Intima-Veränderungen, doch waren 
dieselben auch an den Nierengefässen nur vereinzelt vorhanden, so dass ich dieselben an den Piagefässen vielleicht nicht gefunden habe. Durch diese zweifelhaften Befunde sehe ich mich genöthigt, auf die von Ewald und Gull und Sutton an der Pia mater gemachten Beobachtungen meine Schlüsse zu stützen. Aus den Eingangs angeführten Gründen glaube ich, dass an den Piagefässen in vielen Fällen auch die Intima an der Hypertrophie betheiligt ist; aber auch ohne diese Annahme finden sich die oben gemachten Schlüsse durch die Befunde an den Piagefässen bestätigt. Mit Sicherheit hat Ewald jedenfalls eine Hypertrophie der Gefässwand constatirt, und es besteht daher zwischen den Piagefässen und den Nierenarterien nur insofern ein Unterschied, als bei ersteren vorwiegend die Muscularis, bei letzteren dagegen auch die Intima an dieser Hypertrophie theilnimmt.

Für die Erklärung der Art uod Weise, wie bei Schrumpfniere der erhöhte Blutdruck zu Stande kommt, ist die Thatsache von grosser Bedeutung, dass die Intima-Verdickungen in den kleineren Arterien verhältnissmässig weit stärker sind, als in den etwas grösseren. Die Zahl der neugebildeten elastischen Membranen ist in den Gefässen, die nur eine ganz geringe Muscularis haben, durchschnittlich grösser, als in Arterien mit starker Muscularis. Ist nun die in dieser Arbeit entwickelte Ansicht über die Entstehung der Gefäss-Verdickung richtig, so kann man die heute fast allgemein anerkannte Erklärung der Blutdruck-Steigerung bei Nephritis interstitialis durch einen Krampf der kleineren Arterien nicht aufrechterhalten. Ein solcher könnte eine Drucksteigerung nur in dem vor der verengten Stelle liegenden Gefässbezirk hervorrufen, hinter derselben muss der Blutdruck herabgesetzt sein. Wir müssen also die gesteigerten Widerstände in das Capillarsystem verlegen. $\mathrm{Zu}$ dem gleichen Schluss kommt Ewald auf Grund seiner Untersuchungen. Nur lässt sich gegen seine Behauptang der Einwand erheben, dass die Hypertrophie der Muscularis der Arterien nicht durch eine Drucksteigerung bedingt zu sein braucht, sondern dem dauernden Tonus seine Entstehung verdanken könnte.

Die Vermehrung der Widerstände an irgend einer Stelle des Gefässsystems würde jedoch allein nicht genügen, eine Blutdrucksteigerung zu erzengen. Durch sie würde nur eine Strom- 
Verlangsamung eintreten, wenn nicht das Herz durch Mehrarbeit und Hypertrophie eine normale Strom-Geschwindigkeit erzielte. Vermehrung der Widerstände im Capillarsystem und Herz-Hypertrophie sind also nothwendig zum Zustandekommen der Arterien-Hypertrophie. Zum Beweise dafür möchte ich einen Fall anführen, bei dem sich, wie schon erwähnt, trotz sehr hochgradiger Schrumplung der Nieren, fast gar keine ArterienHypertrophie fand. Das Sections-Protokoll ergab keine Hypertrophie des linken Ventrikels.

Es ist bisher gezeigt worden, dass die bei der Schrumpfniere auftretende Vermehrung der elastischen Membranen als einfache Hypertrophie derselben anzusehen ist. Da aber das elastische Gewebe ein $Z$ wischengewebe darstellt, das an sich keine Vermehrungs-Fähigkeit besitzt, so müssen wir uns die Frage vorlegen, von welchen Gebilden die Entstehung der neuen elastischen Membranen ihren Ausgang nimmt. Ich will mich dabei nicht im Allgemeinen auf die Discussion der Streitfrage einlassen, ob die elastische Faser durch Umwandlung des Zellleibes oder aus dem $Z$ wischengewebe entsteht, sondern lediglich untersuchen, wo die Neubildung der Membranen in der Gefässwand vor sich geht. Natürlich darf man nicht erwarten, in jedem Gefäss die einzelnen Stadien des Processes verfolgen zu können, vielmehr muss man von vornherein aunehmen, dass der Process nach einer gewissen Zeit, wenn nehwlich die BlutdruckSteigerung ihr Maximum erreicht hat, zum Stillstand kommt, und man wird sich dann auch nicht wundern, dass in der grossen Mehrzahl der Arterien nur ausgebildete, vollständig gleich starke Membranen zu sehen sind. Um so wichtiger war es, die vereinzelten Gefässe, in denen der Process offenbar noch im Entstehen begriffen war, zu untersuchen.

Am einfachsten und scheinbar durch das mikroskopische Bild gegeben wäre es, die neuen Membranen durch eine Auffaserung der Elastica interna entstehen zu lassen, und thatsächlich ist auch diese Erklärung von den meisten Autoren angenommen worden. Laughans, Talma, Dmitrijeff und Jores stellten sich den Vorgang in dieser Art vor. Dem gegenüber kann man anführen, dass die Membranen durch Zellthätigkeit wirklich neu gebildet werden, und hat dann die Wahl, sie durch eine 
Zusammenlagerung neugebildeter elastischer Fasern entstehen zu lassen oder eine directe Bildung der Membran durch Zellen anzunehmen. Für alle drei Erklürungs-Arten muss man eine Betheiligung von Zellen in der Intima oder zwischen den Lamellen der Elastica interna annehmen, und wird dadurch auf die in der Literatur schon so viel discutirte Frage gedrängt, woher stammen diese Zellen? Dass dieselben vom Endothel abstammen, glaube ich für die vorliegenden Veränderungen ausschliessen zu können, da ich in mehreren Gefässen das Endothelhäutchen, vollkommen continuirlich von seiner Unterlage abgehoben, im Lumen liegen sah, ohno dass sich irgend ein Zusammenhang mit der innersten Membran durch Zellen oder direct erkennen liess. Wir müssen annehmen, dass Bindegewebszellen, die bei der ursprünglichen Bildung der Elastica interna betheiligt waren, zwischen den Lamellen derselben, oder auch zwischen ihr und dem Endothel, auch später noch vorhanden sind, dass sie aber für gewöbnlich nicht beobachtet werden können, weil ihre dünnen Zellleiber in die Lamellen der elastischen Membran fest eingepresst sind und in dem Schnitt theilweise von denselben bedeckt werden. Vielleicht stehen auch die Räume zwischen den Lamellen durch die Fenster der Membran mit Bindegewebszellen der Media in Verbindung. Diese Zellen werden nun durch den gesteigerten Blutdruck gereizt und nehmen ihre Thätigkeit wieder auf. Sie scheiden zunächst eine homogene Zwischensubstanz aus und drängen dadurch die Lamellen auseinander. Dabei bleibt es jedoch nicht, denn auf diese Weise wäre es nicht zu erklären, dass die Gesammtmasse der elastischen Membranen weit grösser ist, als die der ursprünglichen Elastica interna. Vielmehr ist diese Auseinanderdrängung offenbar nur ein Vorstadium, dem die Bildung neuer elastischer Substanz folgt. Dies war sehr schön an einem Präparat von Fall $1 \mathrm{zu}$ sehen. In den Raum zwischen dem Endothel und der innersten elastischen Haut ragte, auf dem Querschnitt gesehen, ein feines elastisches Häutchen hinein, das continuirlich in eine Lage von Zellen überging, so dass es ganz den Eindruck machte, als ob diese Zellen an ihrer Oberfläche die Membran ausschieden (Fig. 5). Dass die Zellen dabei selbst zum Theil in den neu gebildeten Membranen aufgehen, ist mir unwahrscheinlich, da die in den Maschen zwischen den einzelnen 
Häuten liegenden Zellen stets noch durch ein homogenes Gewebe von den Membranen getrennt sind. Nach dem Gesagten darf man also die Masse der neu gebildeten Membranen nicht, wie es bisher der Einfachheit halber geschen ist, der Intima allein zuschreiben. Ueberhaupt darf man nicht annehmen, dass derartige Processe vor den künstlich gesetzten Grenzen zwischen den drei Arterienhäuten Halt machen. Es ist vielmehr wahrscheinlich, dass in der Media ähnliche Processe verlaufen, um so mehr, als auf diese Weise die schon mehrfach erwähnte Thatsache Erklärung findet, dass die Media über den Stellen, die durch die vermehrten Membranen verdickt waren, vielfach geschwunden .war. Wie aus einzelnen Präparaten zu ersehen war, scheinen sich in dem Bindegewebe zwischen den Muskelbündeln neue Fasern zu bilden, die sich an einander lagern und offenbar die Stelle der späteren Mémbranen einnehmen. Es würde sich hier also nicht um einen wirklichen Schwund der Media, sondern nur um eine Umzäunung der Maskelfasern durch elastische Membranen handeln, wogegen die Form eînzelner, in den Räumen $\mathbf{z w i s c h e n ~ d e n ~ L a m e l l e n ~ g e f u n d e n e r ~ Z e l l e n ~ n i c h t ~}$ spricht. In der Intima hat jedenfalls eine Zusammenlagerung von Fasern zu Membranen nicht stattgefunden, da ich daselbst keine elastischen Fasern beobachtet habe. Etwas: anders gestaltet sich der Process bei dem schon erwähnten 24jährigen Individuúm. Hier wurde offenbar von den erwähnten Zellen der Intima zunächst eine homogene Substanz gebildet, in der dann die elastischen Membranen direct von den Zellen abgeschieden wurden.

Schliesslich möchte ich die gewonnenen Resultate dahin zusammenfassen: Bei allen Formen der chronischen interstitiellen Nephritis treten in den kleineren Arterien des Körpers Veränderungen auf, die im Wesentlichen in einer Hypertrophie der drei Arterienhäute bestehen. Die bisher als Endarteriitis fibrosa bezeichnete IntimaVerdickung besteht nicht aus gewöhnlichem fibrillärem Bindegewebe, sondern aus neugebildeten elastischen Membranen. Daneben findet sich häufig in den kleineren Ärterien auch Arteriosklerose, die sich von der Arterien-Hypertrophie dadurch unterscheidet, dass die Verdickung der Media nicht durch Vermehrung der Muskelfasern, sondern des Bindegewebes bedingt Archiv f. pathol. Auat. Bd. 159. Hft. 3. 
ist. In der Intima findet sich nach innen von den neugebildeten elastischen Membranen eine mehr fibröse oder hyaline Schicht, in der stets Degenerations-Erscheinungen an den elastischen Gewebsbestandtheilen, bisweilen auch Vascularisation und nekrotische Partien vorhanden sind. Beide Veränderungen kommen oft an demselben Gefäss vor, so dass eine scharfe Trennung nicht immer möglich ist. In einer grossen Zahl der Fälle tritt die ArterienHypertrophie dagegen für sich auf. Die Ursache für dieselbe bietet die Blutdracks-Steigerung bei Nephritis interstitialis, und zwar sind die erhöhten Widerstände, durch welche dieselbe bedingt ist, in das Capillarsystem za verlegen.

Die neuen Membranen entstehen durch Auffaserung der Elastica interna und darauffolgende Neubildung, die von den zwischen den Lamellen der Membrana elastica interna gelegenen Zellen ausgeht. Daneben bilden sich wahrscheinlich in der Media elastiche Fasern, die, sich zu Membranen zusammenlagern. -

Von einer Beschreibung aller von mir untersuchten Fälle habe ich abgesehen, um die Arbeit nicht zu sehr auszudehnen; ich werde mich im Folgenden darauf beschränken, einige typische Fälle wiederzugeben. Unter 19 Fällen zeigten 2, wie erwähnt, keine Gefäss-Veränderungen. Unter den übrigen befanden sich zwei Schrumpfnieren, in denen die Gefässe sicher die reine Hypertrophie ohne jede Spur von Arteriosklerose zeigten. In dem einen handelte es sich um einen 38jährigen Mann, im andern um eine noch jugendliche Frau, die an einer Schwangerschafts-Nephritis zu Grunde gegangen war, bei der sich jedoch auch schon interstitielle Processe in der Niere zeigten. Die grosse Mehrzahl der Fälle betraf in den $40 \mathrm{er}$ Jahren oder im Anfang der 50er Jahre stehende Individuen. Auch hier waren meist keine Zeichen von Arteriosklerose vorhanden. Doch zeigten hin und wieder an einzelnen Gefässen die elastischen Membranen nicht mehr ihr sscharfes, contourirtes Aussehen, sondern waren etwas gezackt oder körnig. Bisweilen schien auch die Zwischensubstanz etwas reichlicher zu sein. Bei 3 Individuen, die das 40 ste Jahr bereits überschritten haben, trat die Arteriosklerose entschieden in den Vordergrund und hatte in 2 Fällen zu so starker Verengerung der Gefässe geführt, dass man sie für die Ursache der Nierenschrumpfung ansehen musste. 
In einem Fall fand sich, wie schon erwähnt, trotz hochgradiger Nierenschrumpfung weder Arteriosklerose noch Arterien-Hypertrophie.

Ich werde nun im Folgenden einen Fall von reiner ArterienHypertrophie und einen mit ausgeprägter Arteriosklerose beschreiben, sowie einen dritten, der ein Uebergangs-Stadium $\mathrm{zwischen}$ beiden bezeichnet:

Fall 1.

Männliche Leiche von 38 Jahren.

Krankengeschichte und Sectionsprotokoll sind nicht vorhanden. Die Niere ist von normaler Grösse mit adbärenter Kapsel. Rinde wenig verschmälert. Etwas parenchymatöse Trübung. Aorta ganz normal, hat oben in Folge starker Skoliose eine spitzwinklige Knickung. Herz zeigt Hypertrophie beider Ventrikel, keine Klappen-Veränderungen.

Mikroskopischer Befund: Die Niere bietet in vielen Partieen ein ziemlich normales Ausseben. Stellenweise sind die Epithelien etwas gequollen und unregelmässig begrenzt. Die Gefässe der Marksubstanz sind stark hyperämisch. In der Rinde finden sich vereinzelte Heerde, in denen die Harncanälchen mebr oder weniger zu Grunde gegangen sind. Die Glomeruli in diesen Heerden sind näher an einander gerückt, grösstentheils in kernarme Kugeln verwandelt, und liegen in einem stark kleinzellig infiltrirten Gewebe.

Die Arteriolae rectae sind vielfach garnicht verändert, bei andern, namentlich auch in den Arteriae interlobulares findet sich eine Vermehrung der elastischen Membranen. Im Allgemeinen ist die Verdickung der Wand nicht sehr stark. Die Media ist nach aussen durch eine feine Elastica externa von der Adventitia getrennt, in der sich mässig reichliche elastische Fasern finden. Die Media ist nicht sebr stark verdickt. In ibr finden sich feine elastische Fasern. Die Intima ist nach innen zu durch ein continuirliches Endothelhäutchen abgegrenzt. Zwischen diesem und der innersten elastischen Membran findet sich keine Zellschicht. An einzelnen Stellen liegen übereinander 4 Membranen. Die äusserste ist continuirlich im ganzen Umfang vorhanden. Die inneren gehen vielfach Verbindungen mit ihr. odér untereinander ein. Vielfach enden sie aber auch frei in der Gefässwand. In einem Gefäss spaltet sich von der innersten Lamelle ein dünnes elastisches Hâutchen $a b$, das einer Schicht spindelförmiger Zellen dicht anliegt (Fig. 5). Zwischen den gefalteten Membranen findet sich eine homogene $Z$ wisehensubsanz, in der wenige meist spindelformige Zellen liegen.

Im Allgemeinen ist in diesem Fall die Arterien-Hypertrophie nicht sehr stark entwickelt, was wohl mit der geringen Stärke des Schrumpfungs-Processes in der Niere zusammenhängt. Ich. 
habe jedoch gerade diesen Fall ausgewählt, weil er keine Spur von Artériosklerose zeigte und sich bei ihm die ArterienVeränderungen noch im Entstehen verfolgen liessen.

\section{Fall 2.}

Krankengeschichte und Sectionsbefund dieses Falles sind mir nieht bekannit. Dưrch Herrn Prof. Benda erhielt ich die eine Niere zur Untersuchung und erfuhr von ihm, dass es sich um ein 74 jähriges Individuum handelte. Aorta und Nierenarterie waren sehr stark arteriosklerotisch und atheromatös erkrankt. Die eine Niere war auf die Grösse einer Walnuss zusammengeschrumpft, die andere, von der ich Präparate angefertigt habe, war aucb etwas verkleinert. Die Oberfäche war feinkörnig granulirt, zeigte aber daneben tiefe Einkerbungen und Narben. Rinde verschmälert.

Mikroskopischer Befund: Ueberaus starke Vermehrung des Bindegewebes in Mark und Rinde. Normale Harncanäleben sind nur in sehr geringem Maasse vorhanden. In den meisten sind die Epithelien atrophisch oder platt gegen die Wand gedrückt. Das Lumen ist mit colloiden Massen gefüllt. Glomeruli und Gefässe erscheinen wegen der starken Schrumpfung sehr zahlreich. Fast durchgehends sind die Glomeruli verödet und zu kernarmen Kugeln zusammengeschrumpft.

Die Gefässe sind durchgehends stark verdickt. In den allerkleinsten Arterien ist diese Verdickung durch eine zellig-fibröse Wucherung bedingt. Doch finden sich diese Veränderungen nur an vereinzelten Gefässen. An den meisten besteht eine starke Intima-Verdickung, die wesentlich durch geschichtete elastische Membranen bedingt isi. Dabei ist die Media an einzelnen Arterien ebenfalls verdickt, an anderen vollkommen geschwunden. Daneben finden sich aber, namentlich an den etwas grösseren Gefässen, Veränderungen ganz anderer Art. Die Wand-Verdickung wird so stark, dass sie das Lumen fast vollkommen zum Schwinden bringt. In der Intima findet sich eine starke zellige Wucherung, die bisweilen auch nekrotische Partien aufweist. In der ausseren Schicht der Intima finden sich viele elastische Membranen, die sich, jedoch nach innen zu immer schlechter mit Orcëin färben. Sehliesslich zerfallen sie in einzelne Körnchen und Fäserchen (Fig. 2). Die Media ist ebenfalls in diesen Arterien sehr stark verdickt und zeigt reichliche Vermehrung des Bindegewebes. Vielfach ziehen auch Züge von Fibroblasten von der Adventitia her nach der Intima hin.

\section{Fall 3.}

Weibliche Leiche von 77 Jahren.

Sectionsbefund: Obesitas. Starke Arteriosklerose der Aorta descendens und der Körperarterien. Starke Hypertrophie beider Ventrikel, Dilatation des rechten. Aneurysma racemosum der Milcarterie. Chronische Bronchitis und Emphysem. Adhäsive Pleuritis: Staungs-Induration der Milz. Chronische interstitielle und parenchymatöse Nephritis. Endarteriitis der' 
Nierenarterien. Geringe interstitielle Hepatitis. Arteriosklèrose der Hirnarterien. Beide Nieren kleiner, als gewöhnlich, Kapsel stellenweise adhärent. Oberfäche von fein granulirtem Aussehen. Auf der Schnittläcbe Rinde verschmälert. Lumina der Nierengefässse klaffend.

Mikroskopischer Befund: Starke Vermehrung des Bindegewebes und Atrophie der Harncanälchèn in der Marksubstanz. In der Rinde zahlreiche Stellen, die fast ansschliesslich aus Bindegewebe bestehen, das starke Infiltration und Reste des Nierenparenchyms aufweist. Die Glomeruli sind zum grossen Theil verödet, theils durch Kapsel-Verdickung, theils durch Degeneration der Glomerulus-Sehlingen.

Die Gefässe sind stark verdickt, doch ist das Lumen keineswegs hochgradig verengt. An den grösseren Gefässen sind in der Intima durchschnittlich 4-5 elastische Membranen vorhanden, die jedoch vielfach nicht mehr scharf contourirt sind, sondern ein gezacktes, rosenkranz-ähnliches Aussehen bieten. Die Zwischensubstanz ist reichlicher, als dies bei der reinen Arterien-Hypertrophie der Fall zu sein pflegt. Doeh ist von einer inneren zelligen Schicht nichts zu bemerken. Die Media ist ziemlich stark verdickt und enthält viel Bindegewebe. In der Adventitia findet sich eine mässige Vermehrung der elastischen Fasern. An den kleineren Gefässen ist die Verdickung der Wand im Verhältnis zum Lumen eine weit stärkere, doch scheint letzteres auch hier nicht verengt zu sein. Die Media ist an einzelnen der kleineren Arterien vollkommen geschwunden. Die Wand der allerkleinsten Gefässe ist wiederum durch eine zellig-fibröse Masse verdickt. Neben diesen Veränderungen zeigen andere Gefässe eine starke, aber einfache Hypertrophie ihrer Wandung.

Dièser Fall bildet offenbar den Uebergang von Fall 1 zu Fall 2. An einer grossen Zahl von Arterien sind die WandVerdickungen nur durch die Granularatrophie verursacht. In anderen macht sich dagegen der Einfluss der Alters-Arteriosklerose geltend. Es ist zwar noch zu keiner starken Verengerung der Gefässe durch die Bindegewebs-Wucherung gekommen, so dass man die Arteriosklerose etwa für die Ursache der Nierenschrumpfung ansehen könnte, doch haben sich bereits ZerstörungsProcesse an den elastischen Membranen und Bindegewebs-Vermehrung eingestellt.

Zum Schluss ist es mir eine angenehme Pflicht, allen Herren, die mich durch ihr liebenswürdiges Entgegenkommen unterstützt haben, meinen aufrichtigsten Dank auszusprechen, insbesondere Herm Prof. Benda, der durch die Ueberlassung seines reichen Materials und durch das Interesse, das er meiner Arbeit 
entgegenbrachte, dieselbe wesentlich förderte, und Herrn Geheimrath Arnold, der mir in liebenswürdigster Weise sein Material und einen Platz in seinem Laboratorim zur Verfügung stellte.

\section{Literatur.}

Baumgarten, Ueber chronische Arteriitis und Endarteriitis u. s. w. Dieses Archiv, Bd. 78.

Dmitrijeff, Veränderungen des elastischen Gewebes bei der Arterioklerose. Beiträge v. Ziegler. XXII. 1897.

Ewald; Ueber die Veränderungen kleiner Gefässe bei Morbus Brightii und die darauf bezüglichen Theorien. Dieses Archiv, Bd. 71 .

Friedländer, Arteriitis obliterans. Centralblatt für wissenschaftliche Mediein, 1876, Bd. 55.

Gull und Sutton, Arterio-capillary Fibrosis; Med.-Chir. Transactions. 1872. Johnson, Medico-Chirurgicae Transactions. Vol 29 und 30.

Jores, Neubildung elastischer Fasern bei Endarteritis. Centralblatt f. allgem. Pathologie Bd. VIII.

Langhans, Zur normalen und pathologischen Anatomie der Arterien. Dieses Archiv Bd. 36.

Heubner, Die luische Erkrankung der Hirnarterien. Leipzig, 1874.

Roux, Der Kampf der Theile im Organismus, Leipzig 1881.

Talma, Endarteriitis chronica. Dieses Archiv Bd. 77.

Thoma, Circulationsstörungen bei chronischer Nephritis. Dieses Archiv. Bd. 71.

- Ueber Gefäss- und Bindegewebs-Neubildung in der Arterienwand. Dieses Archiv, Bd. 93, 95, 102, 105 u. 112.

- Lehrbuch der pathologischen Anatomie. Allgemeiner Theil. 1894.

Weissmann und Neumann, Ueber die Veränderungen der elastischen Fasern in Folge von Arteriosklerose. Allgemeine Wiener medic. Zeitung. Bd. 35 .

\section{Erklärung der Abbildungen auf Tafel XIV.}

Fig. 1. Einfache Hypertrophie. Măssige Verdickung der Intima und Media. Das Endothelbäutchen hat sich etwas von der Unterlage abgehoben, ebenso sind die Membranen stellenweise etwas von einander getrennt. Nach We igert und mit Lithioncarmin gefärbtes Präparat. (Zeiss A. Oc. 4. Tubuslänge 140.)

Fig. 2. Arteriosklerotisches Gefäss. In der äusseren Schicht mebrere elastische Membranen, die nach innen zu in einzelne Stücke und Fasern zerfallen. In der innersten Schicht nur eine zellige Wucherung mit hyaliner Zwischensubstanz. Media bis auf eine kleine Stelle ganz geschwunden. Mit Orcëin und Methylenblau gefärbtes Präparat. (Zeiss D. Oc. 2. Tubuslänge 140.) 
Fig. 3. Intima einer hypertrophischen Arterie. Endothel nicht mehr erhalten. Zwischen den Membranen wenige spindelförmige Zellen. Mit Orcëin und Methylenblau gefärbtes Präparat. (Zeiss D. Oc. 4. Tubuslänge 180.)

Fig. 4. Intima einer arteriosklerotischen Arterie. In der äusseren Schicht 2 noch ziemlich elastische Membranen, nach innen zu nur Zerfallsproducte derselben. Starke zellige Wucherung. Die Zellen haben mehr einen polygonalen Charakter. Nach Weigert und mit Lithioncarmin gefärbtes Präparat. (Zeiss D. Oc. 4. Tubuslänge 180.)

Fig. 5. Neubildung der elastischen Membranen in einem hypertropbischen Gefäss. Zwisehen Endothel und innerster Schicht der Elastica interna eine Zellschicht, die einem elastischen Häutchen dicht anliegt. Nach Weigert und mit Lithioncarmin gefärbtes Präparat. (Zeiss D. Oc. 4. Tubuslänge 180.) 